\title{
Le numérique en classe de français pour développer la littératie des adolescents : présentation d'un projet de recherche exploratoire
}

\section{HRONIQUE • Recherche étudiante}

\section{Qu'est le concept de littératie au XXI siècle?}

Depuis une trentaine d'années, la société et l'école occidentales ont été profondément transformées par l'avènement du numérique dans les établissements (Boissière, Fau et Pedró, 2013). L'Internet, en consignant l'information dans des milliards de pages Web, offre à une partie de l'humanité un accès illimité à la plus riche agglomération de connaissances qui n'ait jamais existé. En ce sens, nous serions passés rapidement d'une société industrielle à une société du savoir (Karsenti, 2005) dont l'un des éléments fondamentaux est la numérisation de l'information (Boissière et al., 2013). En réaction à l'évolution numérique de la société, l'école du XXI siècle doit anticiper les enjeux reliés à l'environnement virtuel en vue d'offrir la meilleure formation possible aux jeunes et s'ajuster à ces enjeux (Conseil canadien sur l'apprentissage, 2009). Une des ramifications de la transformation de l'école par le numérique concerne l'apprentissage de la langue maternelle, plus spécifiquement le développement de la littératie des adolescents. Le concept de littératie, autrefois associé au fait d'être instruit et de savoir manier les lettres sur support papier, se complexifie au XXI siècle : il doit désormais inclure l'écriture sur support numérique de telle sorte que certains auteurs parlent en termes de new literacies (Rowsell et Pahl, 2015). D'autres auteurs encore mettent plutôt l'accent sur les différents modes de communication rendus possibles grâce au numérique (Kress, 2014). Enfin, le Programme de formation de l'école québécoise indique que l'enseignant a le devoir de mettre les technologies à la disposition des élèves pour développer leur compétence en 
français (Ministère de l'Éducation, du Loisir et du Sport, 2009) sans toutefois offrir beaucoup de détails sur les façons les plus efficaces de les utiliser à cette fin.

\section{Comment exploiter le numérique en classe de français de manière optimale?}

À ce jour, l'outil technologique le plus documenté est le traitement de texte. Plusieurs méta-analyses ont démontré les apports bénéfiques de l'utilisation du traitement de texte sur le développement de la compétence à écrire des élèves. Il en ressort que non seulement les élèves qui écrivent au traitement de texte sont plus engagés à la tâche, mais ils produisent aussi un travail écrit plus long et de meilleure qualité que ceux qui écrivent à la main. Certains chercheurs recommandent l'utilisation du traitement de texte comme outil principal d'apprentissage de l'écriture (Graham et Perin, 2007). Au Québec, les auteurs d'une enquête sur les classes-portables (des classes où chacun des élèves dispose d'un ordinateur portable) ont spécifié que l'écriture au traitement de texte est surtout plus flexible et rapide, ce qui permet aux élèves de penser davantage au contenu de leur texte et de réviser plus longtemps (Karsenti et al., 2012). Comment exploiter au maximum le support numérique en classe de français? Plusieurs pistes sont récemment explorées telles que l'utilisation judicieuse d'outils de correction et de référence linguistique complets, comme Antidote (Ouellet, 2013), d'outils de correction audiovisuelle (Cabot et Lévesque, 2015), d'outils de collaboration comme Google Drive (Cotugno, 2014), etc.

\section{Un projet de recherche exploratoire auprès d'enseignants de français du Québec}

Face à cet éventail de potentialités, nous nous sommes demandé quelles seraient les façons optimales d'exploiter le numérique pour développer la littératie des élèves en classe de français du secondaire dans le contexte d'une tâche de lecture et d'écriture. Pour répondre à cette question, nous avons piloté une étude qualitative exploratoire auprès d'enseignants de français d'écoles secondaires du Québec. Nous avons utilisé des questionnaires en ligne destinés aux enseignants $(\mathrm{N}=40)$, des entretiens individuels avec des enseignants volontaires $(\mathrm{N}=13)$ et des observations en classe d'enseignants d'écoles privées du Québec $(\mathrm{N}=3)$. Enfin, un questionnaire en ligne a été rempli par les élèves des trois enseignants observés $(\mathrm{N}=114)$. Les grands thèmes de notre collecte de données sont étroitement liés aux trois grands objectifs de notre projet, soit: définir le concept de «nouvelle " littératie; définir et mieux comprendre comment le numérique en classe de français du secondaire est susceptible de développer la littératie des élèves dans son volet concernant la lecture et dans son volet concernant l'écriture.

\section{En guise de conclusion}

À travers cette étude exploratoire, nous souhaitons proposer une définition du concept de littératie de même qu'un modèle de compréhension d'une littératie « nouvelle » afin de mieux anticiper les façons optimales d'utiliser les technologies en classe de français. Nous croyons que les technologies ont le potentiel de catalyser une maitrise du français telle qu'il serait éventuellement possible de proposer, dans une optique similaire à celle des réclamations de la Fédération des établissements d'enseignement privés (Dion-Viens, 2016), un programme d'enseignement de cette matière sur quatre ans plutôt que cinq. 


\section{Références}

Boissière, J., Fau, S. et Pedró, F. (2013). Le numérique : une chance pour l'école. Paris : Armand Colin.

Cabot, I. et Lévesque, M.-C. (2015). La correction audiovidéo : une pratique profitable?. Pédagogie collégiale, 28(3), 10-15. Repéré à http://aqpc.qc.ca/sites/default/files/revue/CabotLevesque-Vol 28-3.pdf

Conseil canadien sur l'apprentissage. (2009). État de l'apprentissage virtuel au Canada. Repéré à http://www.bdaa.ca/biblio/recherche/cca/virtuel/virtuel.pdf

Cotugno, M. (2014). Using Google Drive to prepare students for workplace writing and to encourage student responsibility, collaboration, and revision. Teaching English in the Two Year College, 42(1), 65-76.

Dion-Viens, D. (2016, 15 novembre). Terminer son secondaire en quatre ans : La Fédération des écoles privées réclame plus de flexibilité. Le Journal de Québec. Repéré à

http://www.journaldequebec.com/2016/11/15/completer-son-secondaire-en-quatre-ans

Graham, S. et Perin, D. (2007). A meta-analysis of writing instruction for adolescent students. Journal of Educational Psychology, 99(3), 445-476. http://dx.doi.org/10.1037/0022-0663.99.3.445

Karsenti, T. (2005). Les technologies de l'information et de la communication dans la pédagogie. Dans C. Gauthier et M. Tardif (dir.), La pédagogie : théories et pratiques de l’Antiquité à nos jours (2éd., p. 255-273). Montréal, QC : Gaëtan Morin Éditeur.

Karsenti, T., Collin, S., Dupuis, A., Villeneuve, S., Dumouchel, G. et Robin, J.-P. (2012). Avantages et défis inhérents à l'usage des ordinateurs portables au primaire et au secondaire: Résultats de la $2^{e}$ enquête auprès de la Commission scolaire Eastern Townships. Montréal, QC : CRIFPE.

Kress, G. (2014). What is mode?. Dans C. Jewitt (dir.), The Routledge handbook of multimodal analysis (2 éd., p. 54-67). Oxon : Routledge.

Ministère de l'Éducation, du Loisir et du Sport. (2009). Programme de formation de l'école québécoise, Enseignement secondaire, deuxième cycle, Français langue d'enseignement. Repéré à http://www1.education.gouv.qc.ca/sections/ programmeFormation/secondaire2/medias/PFEQ FrancaisLangueEnseignement.pdf

Ouellet, M. (2013). Mesure et évaluation des apports d'un correcticiel : rapport de recherche. Repéré à https://cdc.qc.ca/ parea/788538-ouellet-mesure-evaluation-apports-correcticiel-drummondville-PAREA-2013.pdf

Rowsell, J. et Pahl, K. (2015). The Routledge handbook of literacy studies. Londres : Routledge Taylor \& Francis Group.

\section{Pour citer cet article}

Dupuis, A. (2017). Le numérique en classe de français pour développer la littératie des adolescents : présentation d'un projet de recherche exploratoire. Formation et profession, 25(2), 115-117.

http://dx.doi.org/10.18162/fp.2017.a130 